\title{
Myelin-Associated Glycoprotein Is a Myelin Signal that Modulates the Caliber of Myelinated Axons
}

\author{
Xinghua Yin, ${ }^{1}$ Thomas O. Crawford, ${ }^{2}$ John W. Griffin, ${ }^{2,3}$ Pang-hsien Tu, ${ }^{4}$ Virginia M.-Y. Lee, ${ }^{4}$ Chumei Li, ${ }^{5}$ \\ John Roder, ${ }^{5}$ and Bruce D. Trapp ${ }^{1}$ \\ ${ }^{1}$ Department of Neurosciences, Lerner Research Institute, The Cleveland Clinic Foundation, Cleveland, Ohio 44195, \\ Departments of ${ }^{2}$ Neurology and ${ }^{3}$ Neurosciences, Johns Hopkins University School of Medicine, Baltimore, Maryland \\ 21287, ${ }^{4}$ Department of Pathology and Laboratory Medicine, Division of Anatomic Pathology, University of Pennsylvania \\ School of Medicine, Philadelphia, Pennsylvania 19104, and 5Samuel Lunenfeld Research Institute, Mt. Sinai Hospital, \\ Toronto, Ontario M5G 1X5 Canada
}

Myelination increases neuronal conduction velocity through its insulating properties and an unidentified extrinsic effect that increases axonal caliber. Although it is well established that demyelination can cause axonal atrophy, the myelin molecule that regulates axonal caliber is not known. Loss of the structural proteins of compact peripheral nervous system (PNS) myelin, $\mathrm{P}_{\mathrm{O}}$ protein, and myelin basic protein does not lead to axonal atrophy. This study demonstrates that mice with a null mutation of the myelin-associated glycoprotein (MAG) gene have a chronic atrophy of myelinated PNS axons that results in paranodal myelin tomaculi and axonal degeneration. Absence of
MAG was correlated with reduced axonal calibers, decreased neurofilament spacing, and reduced neurofilament phosphorylation. Because axonal atrophy and degeneration in MAGdeficient mice occur in the absence of inflammation, hypomyelination, significant demyelination-remyelination, or gain of function mutations, these data support a functional role for MAG in modulating the maturation and viability of myelinated axons.

Key words: myelin-associated glycoprotein; axonal atrophy; neurofilament phosphorylation; axonal caliber; neurofilament spacing; paranodal tomaculi
Peripheral nerve axons are ensheathed by Schwann cells. Axons with larger calibers are surrounded by myelin, which is a multilayered, tightly compacted extension of the Schwann cell plasma membrane. Myelination is essential for rapid propagation of action potentials and for normal neurological function (Waxman, 1980). Unmyelinated axons, usually of smaller caliber, are ensheathed by single uncompacted Schwann cell membranes. Myelination of peripheral nervous system (PNS) axons is initiated by an axonally derived signal (Aguayo et al., 1976; Weinberg and Spencer, 1976), and in most settings maintenance of PNS myelin internodes depends on physical contact with a viable axon (Griffin et al., 1993). Although axonal regulation of Schwann cell phenotype is well established, it is also clear that myelination influences the axon locally. Myelination is associated with increases in axonal caliber (Windebank et al., 1985). This effect is important functionally because axonal caliber is a determinant of neuronal conduction velocity (Arbuthnott et al., 1980). Axonal caliber is influenced by neurofilament number (Hoffman and Griffin, 1992; Ohara et al., 1993; Eyer and Peterson, 1994) and neurofilament spacing (Friede and Samorajski, 1971; Hoffman and Griffin, 1992).

The extrinsic effect of myelination on axonal caliber was demonstrated in developing rat optic nerve (Sanchez et al., 1996), and by comparing axonal caliber in myelinated regions with the non-

Received Nov. 10, 1997; revised Dec. 22, 1997; accepted Dec. 29, 1997.

This work was supported by National Institutes of Health Grant NS29818. We thank Karen Toil for typing this manuscript, and Robert Wesley for technical assistance.

Correspondence should be addressed to Dr. Bruce Trapp, Department of Neurosciences/NC30, Lerner Research Institute, The Cleveland Clinic Foundation, 9500 Euclid Avenue, Cleveland, OH 44195.

Copyright (C) 1998 Society for Neuroscience $0270-6474 / 98 / 181953-10 \$ 05.00 / 0$ myelinated initial segment of dorsal root ganglion axons (Hsieh et al., 1994). The trophic effect of myelination on axonal caliber is also reversible. In demyelinated internodes of the Trembler mouse, axonal calibers were smaller, and neurofilaments (NFs) were spaced closer and were less phosphorylated than those in control mice (deWaegh et al., 1992). Reduced axonal calibers and neurofilament spacing after demyelination is also reversed by remyelination (Hsieh et al., 1993).

Neurofilaments consist of three subunits with apparent molecular weights of 68,150 , and $200 \mathrm{kDa}$ that are referred to as NFL, NFM, and NFH, respectively (Hoffman and Lasek, 1975; Hirokawa et al., 1984). All subunits share a highly conserved central rod domain but differ in their $\mathrm{C}$-terminal region. $\mathrm{NFL}$ forms the core of the $10 \mathrm{~nm}$ neurofilaments, and the C-terminal regions of $\mathrm{NFH}$ and $\mathrm{NFM}$ include sidearms that extend from the core filament. The C-terminal region of NFH and NFM contains 40-50 and 10-15 lysine-serine-proline (KSP) repeats, respectively, which are potential phosphorylation sites. It has been proposed that phosphorylation of NFH and NFM increases the total negative charge and the lateral extension of neurofilament sidearms (Hirokawa et al., 1984; Nakagawa et al., 1995). Sidearm extension, in turn, increases neurofilament spacing and axonal caliber.

The studies discussed above support a positive correlation between myelination, neurofilament number, neurofilament spacing, neurofilament phosphorylation, and axonal caliber. Although the mechanism by which myelination regulates axonal cytoskeleton is unclear, one tenable possibility is that a molecule(s) in the adaxonal membrane of the myelin internode regulates neurofilament number or neurofilament phosphorylation or both. The following observations support this hypoth- 
esis. Ensheathment of axons by oligodendrocyte processes that do not form myelin because of mutations in myelin protein genes still increase axonal caliber and neurofilament spacing (Sanchez et al., 1996). Increased axonal calibers, therefore, can occur in the absence of myelination as long as the axon is ensheathed by a myelin-forming cell. However, axonal ensheathment by Schwann cells does not guarantee increased axonal caliber of unmyelinated fibers, suggesting that a molecule enriched in the adaxonal membrane of myelinating Schwann cells and absent from the adaxonal membrane of nonmyelinating Schwann cells regulates axonal caliber.

Although little is known about the molecules that regulate the myelinotropic effect on axonal cytoskeleton, the myelinassociated glycoprotein (MAG) is an attractive candidate. MAG is a member of the I-type lectin subgroup of the Ig gene superfamily (Arquint et al., 1987; Lai et al., 1987; Salzer et al., 1987) and is expressed exclusively by myelinating cells where it is enriched in the adaxonal membrane of the myelin internode (Sternberger et al., 1979; Trapp and Quarles, 1982; Trapp et al., 1989). MAG was initially considered a potential receptor for an axonal ligand important to the initiation and progression of myelination. This was based on the biochemical properties of MAG and enrichment in periaxonal membranes of myelinated fibers and by studies of transfected Schwann cells in vitro that correlated MAG overexpression with accelerated myelination (Owens et al., 1990) and MAG underexpression with hypomyelination (Owens and Bunge, 1991). Normal myelination in MAGdeficient mice (Li et al., 1994; Montag et al., 1994), however, has ruled out an essential role for MAG in myelin formation in vivo. In vitro, neurite outgrowth can be inhibited by MAG (McKerracher et al., 1994; Mukhopadhyay et al., 1994), and MAG-coated beads can cause growth cone collapse (Li et al., 1996). These data raised the possibility that MAG directly or indirectly modulates axonal cytoskeleton. This study investigated whether alterations in axonal cytoskeleton occur in MAGdeficient myelinated fibers. The results, for the first time, implicate MAG in regulating the levels of neurofilament spacing and axonal caliber in mature myelinated axons. The data are consistent with the hypothesis that MAG exerts a major extrinsic influence on the organization of the axonal cytoskeleton and the viability of axons.

\section{MATERIALS AND METHODS}

Morphological analysis and morphometrics. Mice deficient in MAG at the mRNA and protein levels (Li et al., 1994) and age-matched controls were perfused with $4 \%$ paraformaldehyde, $2.5 \%$ glutaraldehyde, and $0.08 \mathrm{M}$ of Sorrenson's buffer, post-fixed in $\mathrm{O}_{\mathrm{S}} \mathrm{O}_{4}$, and embedded in Epon. For light microscopy, $1-\mu \mathrm{m}$-thick sections were stained with toluidine blue. For electron microscopy, approximately 120 -nm-thick sections were stained with uranyl acetate and lead citrate, and were examined in a Philips CM100 electron microscope. Axonal caliber of 35-d-old fibers was determined by analysis of electron micrographs (final magnification $3000 \times$ ) with the aid of a computer-based morphometric system, as described previously (Xu et al., 1996). For 3-, 6-, 9-, and 16-month-old nerves, similar analysis was performed on 1- $\mu$ m-thick Epon sections.

Axonal caliber. Mean axon calibers of myelinated fibers were obtained from 1- $\mu$ m-thick Epon sections of sciatic nerve as described previously (Wong et al., 1995). Continuous, nonoverlapping images in regions of good cross-sectional orientation containing $\sim 1000$ axons were digitized with a standard video frame grabber (Targa M8). The optimal gray scale and lighting were determined for each image individually, and all axons with a cross-sectional area $>1.5 \mu \mathrm{m}^{2}$ centered within the frame were enumerated with a computerized morphometric program (Bioquant MEGx, R \& M Biometrics, Memphis, TN). Axonal caliber is determined by the diameter of a circle of area equivalent to each axon. Four nerves from control and MAG-deficient mice were analyzed at 3, 6, 9, and 16 months of age. To combine different age groups for statistical comparison, the average axonal diameter of each nerve was transformed by subtracting the appropriate age control group mean diameter value. Statistical analysis was performed by the two-tailed Student's $t$ test.

Neurofilament spacing. Nearest neighbor distance was used to measure neurofilament spacing. Electron micrographs were illuminated on a view box and imaged with a video camera. Neurofilament bundles that were oriented in true transverse section were accepted and digitized with Bioquant software ( $\mathrm{R} \& \mathrm{M}$ Biometrics). Nearest neighbor distance was determined as described previously (Xu et al., 1996). For statistical analysis, the mean neurofilament nearest neighbor distance for 11 or more axons for each age was compared by the two-tailed Student's $t$ test.

Neurofilament content and phosphorylation. Three pairs of sciatic nerves from 35-d-old, 3-month-old, and 9-month-old MAG-deficient and age-matched wild-type mice were used for quantitative Western blot analysis of NF proteins with a panel of NF-specific mouse monoclonal antibodies (mAbs) and rabbit polyclonal antisera. These studies were performed as described previously ( $\mathrm{Tu}$ et al., 1995), with minor modifications. Briefly, sciatic nerves were homogenized, sonicated in $200 \mu \mathrm{l}$ of BUST buffer (50 mM Tris-HC1, pH 7.4, $8 \mathrm{M}$ urea, $2 \% \beta$-mercaptoethanol, and $0.5 \% \mathrm{SDS}$ ), and centrifuged at $40 \times 10^{3} \mathrm{rpm}$, at $25^{\circ} \mathrm{C}$ for $30 \mathrm{~min}$. Protein concentrations in the supernatants were determined using Coomassie protein assay according to the manufacturer's instructions. Nerves from three separate animals were analyzed at each time point. Each sample was loaded in triplicate, and each lane contained $10 \mu \mathrm{g}$ of total protein. Blots were then cut into three parts for analyses. The top third was incubated overnight with RM024 (a mAb specific for highly phosphorylated NFH, NFHP +++ ), Rmd09 (a mAb specific for the poorly or nonphosphorylated NFH, NFHP-), or rabbit polyclonal antiserum raised against the last 20 amino acids of $\mathrm{NFH}$ for total $\mathrm{NFH}$. The middle third was probed with RM0189 (a mAb for rod domain of NFM) for total NFM level or with RM055 (a mAb specific for highly phosphorylated NFM, NFMP +++ ). Finally, the lower third was probed with rabbit polyclonal antiserum raised against the C-terminal 20 amino acids of NFL for total NFL. The blots were incubated for $1 \mathrm{hr}$ with $10 \mu \mathrm{Ci}^{125} \mathrm{I}$ conjugated goat anti-mouse IgG for the mouse mAbs (RM024, Rmd09, RM055, and RM0189) or ${ }^{125}$ I-conjugated protein A for the rabbit antiNFH and anti-NFL polyclonal antisera. The dried blots were exposed to phosphorimager plates for various time periods, and individual bands were visualized and quantified with ImageQuant software (Molecular Dynamics, Sunnyvale, CA). Statistical analysis was performed by the Student's $t$ test.

Electron microscopic immunocytochemistry. Segments of sciatic nerve from control and 9-month-old MAG-deficient mice were fixed in $2.5 \%$ glutaraldehyde and $4 \%$ paraformaldehyde, infiltrated with $2.3 \mathrm{M}$ sucrose and $30 \%$ polyvinylpyrrolidone, placed on specimen stubs, and frozen in liquid nitrogen. Ultrathin cryostections $(\sim 120$-nm-thick) were cut on glass knives in an ultracryomicrotome (Ultracut S, Reichert Scientific Instruments) maintained at $-100^{\circ} \mathrm{C}$. The sections were placed on carbonand formvar-coated grids and immunostained by previously described immunogold procedures (Trapp et al., 1989). After application of primary antibodies and gold-labeled secondary antibodies, the sections were placed on $2.5 \%$ glutaraldehyde for $15 \mathrm{~min}$, stained with uranyl acetate, and mounted in $1.5 \%$ methycellulose containing $3.0 \%$ uranyl acetate. Immunostained sections were examined in a Philips CM100 electron microscope. The antibodies were the same as those used in Western blots and include RM024 (anti-NFHP +++ ; 1:5000 dilution) and Rmd09 (anti-NFHP-; 1:250 dilution). The number of gold particles per area of axoplasm was quantified on electron micrographs printed at magnification of $28,500 \times$. Axoplasm $\left(21 \mathrm{\mu m}^{2}\right)$ from 15 control and $15 \mathrm{MAG}-$ deficient fibers was analyzed. Sampling was restricted to axons surrounded by normal-appearing myelin containing 53-67 lamellae. Statistical analysis was performed by the Student's $t$ test.

Teased fiber preparations. Glutaraldehyde-fixed sciatic nerves from 3and 9-month-old control and MAG-deficient mice were osmicated and placed in glycerol. Individual fibers were removed with forceps, placed on slides, and analyzed with a Zeiss axiophot microscope. Thick myelin segments were quantified and scored as to paranodal or internodal location. Statistical analysis was performed by the Student's $t$ test. 


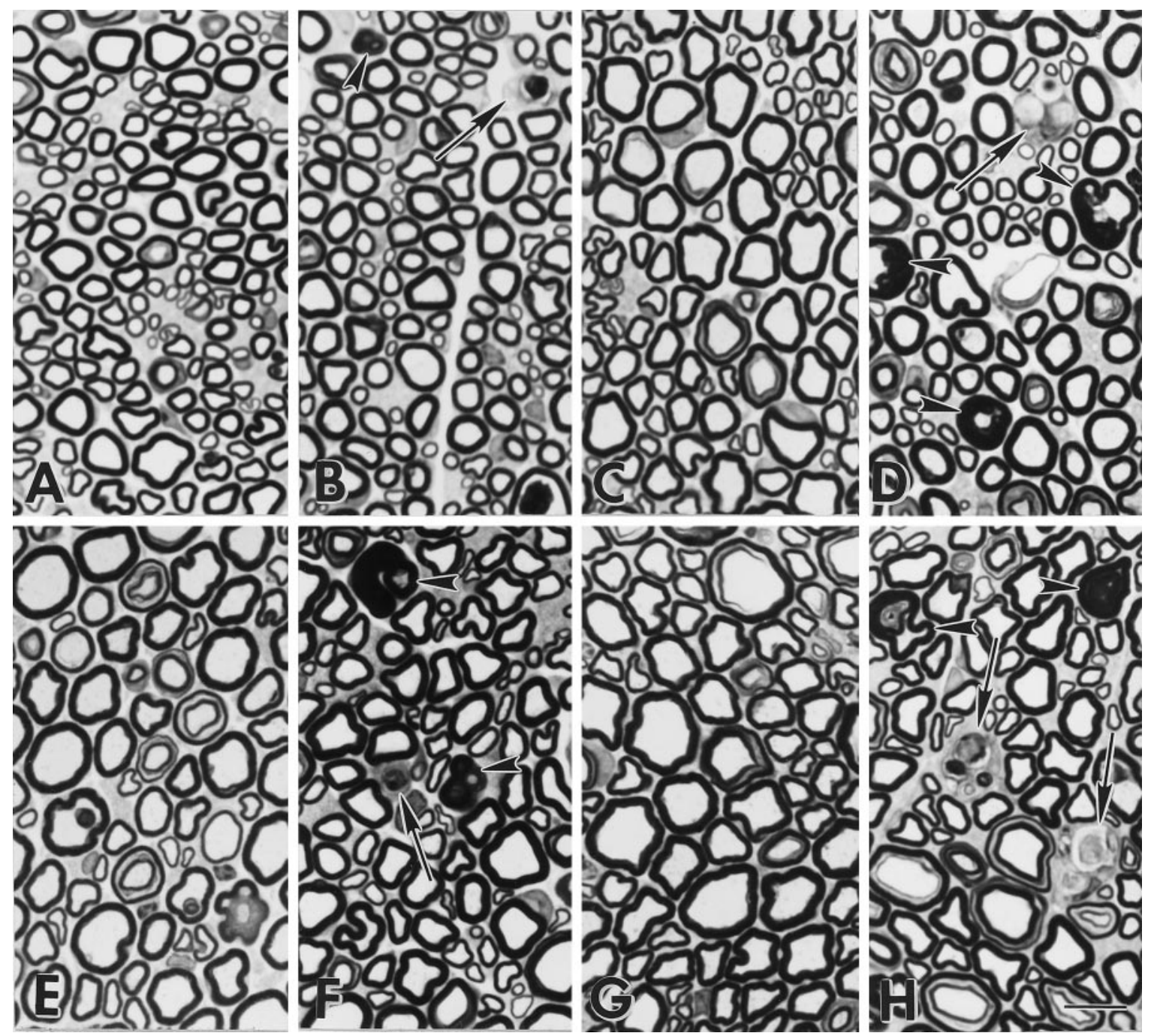

Figure 1. MAG-deficient sciatic nerves contain degenerating myelin internodes and redundant myelin. $A-D$, Epon sections of sciatic nerves from P35 $(A=\mathrm{MAG}+/+, B=\mathrm{MAG}-/-)$ and 3-month-old mice $(C=\mathrm{MAG}+/+, D=\mathrm{MAG}-/-)$. Occasional MAG-deficient myelinated fibers are degenerating $(B, D$, arrows $)$, and some axons are surrounded by redundant or excessive myelin $(B, D$, arrowheads $) . E-H, A t 9$ months $(E=\mathrm{MAG}+/+$, $F=\mathrm{MAG}-/-)$ and 16 months $(G=\mathrm{MAG}+/+, H=\mathrm{MAG}-/-)$ of age, degenerating myelin internodes (arrows) and redundant myelin (arrowheads) persist in MAG-deficient sciatic nerves. Compared with control nerves, myelinated fibers in MAG-deficient nerves appear to have reduced axonal calibers. Scale bar, $10 \mu \mathrm{m}$.

\section{RESULTS}

To study pathological changes in MAG-deficient myelinated fibers, the light microscopic appearance of sciatic nerves from control and MAG-deficient mice was compared. Epon sections (1- $\mu$ m-thick) of sciatic nerves from postnatal day (P) 7, P14, and P35, and 3-, 6-, 9- and 16-month-old mice were studied. The appearance of myelinated fibers in sections from 7- and 14-d-old MAG-deficient sciatic nerves did not differ significantly from age-matched control nerves (data not shown). At P35 (Fig. $1 A, B)$, sections of MAG-deficient nerves contained two to three profiles with classic features of Wallerian degeneration: multiple myelin ovoids, no axon, and scattered macrophages. Sections from 3-month-old MAG-deficient mice contained four to five fibers undergoing Wallerian-like degeneration, redundant or asymmetrically positioned myelin, and an apparent reduction in the caliber of myelinated axons (Fig. $1 C, D$ ). Sections from 9- and 16-month-old MAG-deficient nerves contained a greater proportion of redundant myelin profiles, four to nine degenerating myelinated fibers, and an apparent decrease in the calibers of myelinated axons (Fig. $1 E-H)$. Degenerating myelinated fibers were detected in control nerves at 16 months of age and averaged less than one per section. Compared with control sciatic nerves, Student's $t$ test analysis detected a statistically significant increase in the incidence of Wallerian degeneration in P35 and older MAG-deficient nerves (Table 1). These observations extend previous reports of axonal pathology and Wallerian degeneration in adult MAG-deficient nerves (Fruttiger et al., 1995; Carenini et al., 1997), and they raise the possibility that myelinated fibers in MAG-deficient mice have reduced axonal calibers.

To test whether a generalized reduction in axonal caliber occurred in MAG-deficient sciatic nerves, a mean average axonal caliber of $\sim 1000$ myelinated fibers from four control and four MAG-deficient mice was obtained at P35 and 3, 6, 9, and 16 months of age. Axonal calibers in 35-d-old MAG-deficient and control nerves were similar (data not shown). By 3 months and thereafter, myelinated axons in MAG-deficient nerves were significantly smaller (all ages combined, $p<0.005$ ) than control axons (Fig. 2). 
Table 1. Quantitative analysis of Wallerian degeneration in sciatic nerve of MAG-deficient and control mice

\begin{tabular}{lllllll} 
& \multicolumn{1}{l}{ Age } & & \\
\cline { 2 - 6 } & 7 and $14 \mathrm{~d}$ & $35 \mathrm{~d}$ & 3 month & 6 month & 9 month & 0 \\
$\mathrm{MAG}+1+$ & 0 & 0 & 0 & 0 & $0.5 \pm 0.6$ \\
$\mathrm{MAG}-1-$ & 0 & $2.0 \pm 0.8^{*}$ & $4.2 \pm 1.3^{*}$ & $4.3 \pm 0.5^{* *}$ & $5.3 \pm 1.5^{* *}$ & $5.4 \pm 2.7^{*}$ \\
\hline
\end{tabular}

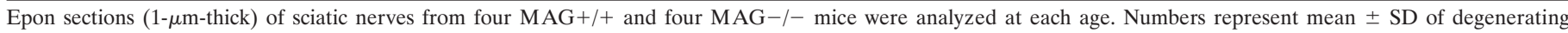
myelinated internodes per total nerve cross section.

${ }^{*} p<0.01 ; *{ }^{*} p<0.001$.

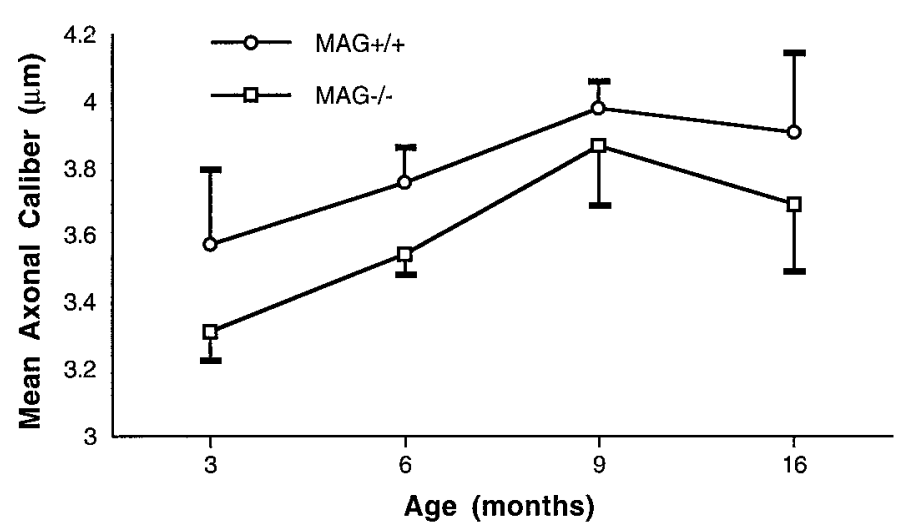

Figure 2. Average axonal calibers of myelinated fibers in control and MAG-deficient sciatic nerves. MAG-deficient myelinated axons have reduced calibers. Axons surrounded by normal-appearing myelin in MAG-deficient nerves are significantly smaller than those in control nerves ( $p<0.005$, for all ages combined). Statistical analysis was performed by the two-tailed Student's $t$ test. Values are mean $\pm \mathrm{SD}$.

\section{Interneurofilament spacing is reduced in MAG-deficient mice}

Because decreased axonal caliber can correlate with decreased neurofilament spacing (deWaegh et al., 1992; Hsieh et al., 1994; Sanchez et al., 1996), the nearest neighbor neurofilament distance was compared in myelinated axons from MAG-deficient and control mice. Analysis was restricted to those axons surrounded by normal-appearing myelin. In cross sections of myelinated axons, neurofilaments appear as 10-nm-thick dots (Fig. 3A, arrowheads) from which thread-like sidearms extend (Fig. $3 A$, arrows). The spacings between neurofilaments appear greater in control axons (Fig. $3 A$ ) than in MAG-deficient axons (Fig. 3B). Myelinated axons were sampled randomly in electron micrographs of 14and 35-d-old and 3- and 9-month-old MAG-deficient and control sciatic nerves. Between 1420 and 3930 neurofilaments were identified, and the distance to their nearest neighbor was measured. Neurofilament spacing was similar in MAG-deficient and wildtype myelinated axons at P14 (data not shown). Statistically significant differences in the nearest neighbor neurofilament distance in MAG-deficient and control nerves were detected at P35, 3 months, and 9 months of age (Fig. $3 C-E$ ).

MAG is not present in the adaxonal Schwann cell membrane of unmyelinated fibers (Trapp et al., 1989). If alterations in neurofilament spacing are regulated by $\mathrm{MAG}$, then the nearest neighbor neurofilament distance in unmyelinated axons should be identical in MAG-deficient and control mice. Therefore, $\sim 950$ neurofilaments in unmyelinated axons from 9-month-old MAGdeficient and control mice were identified, and the distance to their nearest neighbor was measured. The spacing of neurofilaments in unmyelinated fibers was identical in MAG-deficient and control mice (Fig. 3F). These studies correlated reduced neurofilament spacing with the loss of MAG in the adaxonal Schwann cell membrane.

\section{MAG-deficient nerves have decreased neurofilament phosphorylation}

Alterations in axonal diameter and neurofilament spacing have been correlated with the phosphorylation state of $\mathrm{NFH}$ and NFM (deWaegh et al., 1992; Tu et al., 1995) and with alterations in NF subunit ratios (Tu et al., 1995; Xu et al., 1996). Based on these observations and the data in Figures 1-3, the neurofilament subunit composition and the extent of neurofilament protein phosphorylation were compared in sciatic nerve homogenates from MAG-deficient and wild-type mice. Significant decreases in the levels of total NFL and NFH were detected in MAGdeficient nerve homogenates from P35, 3- and 9-month-old mice, whereas levels of total NFM were comparable to those in the age-matched control nerves (Fig. 4). In the absence of MAG, therefore, NF subunit ratios are altered. Relative levels of NFH and NFM phosphorylation $(\mathrm{P}+++)$ were also significantly decreased in the MAG-deficient nerves. The reduction in $\mathrm{NFHP}+++$ paralleled that of total $\mathrm{NFH}$, but there was no decrease in nonphosphorylated $(\mathrm{P}-) \mathrm{NFH}$ epitopes in 3- and 9-month-old MAG-deficient nerves. These observations indicate reduced NFH phosphorylation levels in the MAG-deficient mice relative to NFH nonphosphorylated epitopes. Although total NFM remained unchanged in MAG-deficient nerves, a significant reduction in NFM phosphorylation was detected in 3- and 9-month-old nerves (Fig. 4). NFM is also a structural component of cross-bridges, and these cross-bridges can control the spacing of neurofilaments (Nakagawa et al., 1995).

The $20-30 \%$ decrease in the amount of highly phosphorylated NFM and NFH in MAG-deficient mice is a minimum estimate because the homogenates of sciatic nerve contained both myelinated and unmyelinated axons, and our electron microscopy data showed that the NFs in the unmyelinated axons remained unchanged (Fig. $3 F$ ). To overcome these possible limitations and to analyze NFH phosphorylation states in myelinated axons, ultrathin cryosections of adult sciatic nerves were immunostained with $\mathrm{NFHP}+++$ and NFHP - antibodies using immunogold procedures (Fig. $4 C, D$ ). Gold particles per unit area of axoplasm were quantified in axons surrounded by 53-67 normal-appearing myelin lamellae. When compared with control fibers, a $50 \%$ reduction in NFHP +++ epitope labeling and a fivefold increase in NFHP - epitope labeling was detected in MAG-deficient fibers (Fig. 4E). Because neurofilament density is higher in MAGdeficient fibers, analysis of these NF epitopes per unit area of axoplasm indicates significant reductions in NFH phosphorylation in myelinated axons of MAG-deficient nerves. 

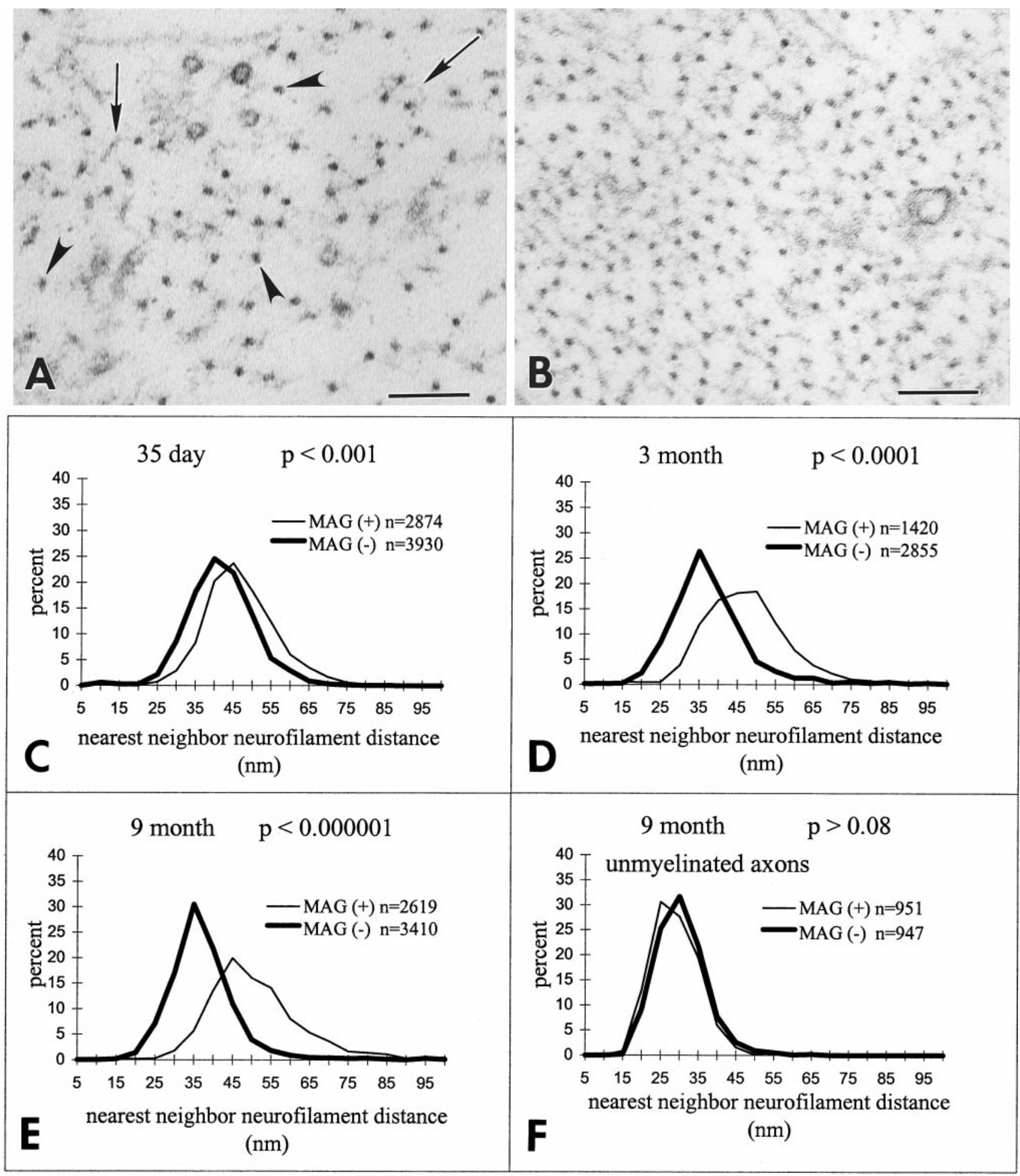

Figure 3. MAG-deficient nerves have reduced neurofilament spacing. $A, B$, In electron micrographs, neurofilaments (arrowheads) appear as 10-nm-thick structures from which sidearms (arrows) extend. Compared with myelinated axons in 9-month-old control nerves $(A)$, neurofilaments are spaced closer together in 9-month-old MAG-deficient myelinated axons $(B)$. Scale bar, $0.1 \mu \mathrm{m}$. $C-E$, Compared with myelinated axons in control nerves, the neurofilament nearest neighbor distance in myelinated axons in MAG-deficient nerves was significantly reduced at P35 $(C), 3$ months $(D)$, and 9 months $(E)$. Statistical analysis by Student's $t$ test analysis; $n=$ number of neurofilaments analyzed. $F$, Neurofilament spacing in unmyelinated axons was similar in control and MAG-deficient nerves from 9-month-old mice; $n=$ number of neurofilaments analyzed.

\section{Collapse of paranodal axons and formation of} redundant myelin

A significant number of myelinated fibers in transverse sections from adult MAG-deficient sciatic nerves had excessive myelin thickness (Fig. 1). The extent and location of thick myelin was studied in teased fibers from control and MAG-deficient sciatic nerves. Thick myelin segments were quantified in 85 and 150 internodes from 3- and 9-month-old mice, respectively (Fig. 5). 


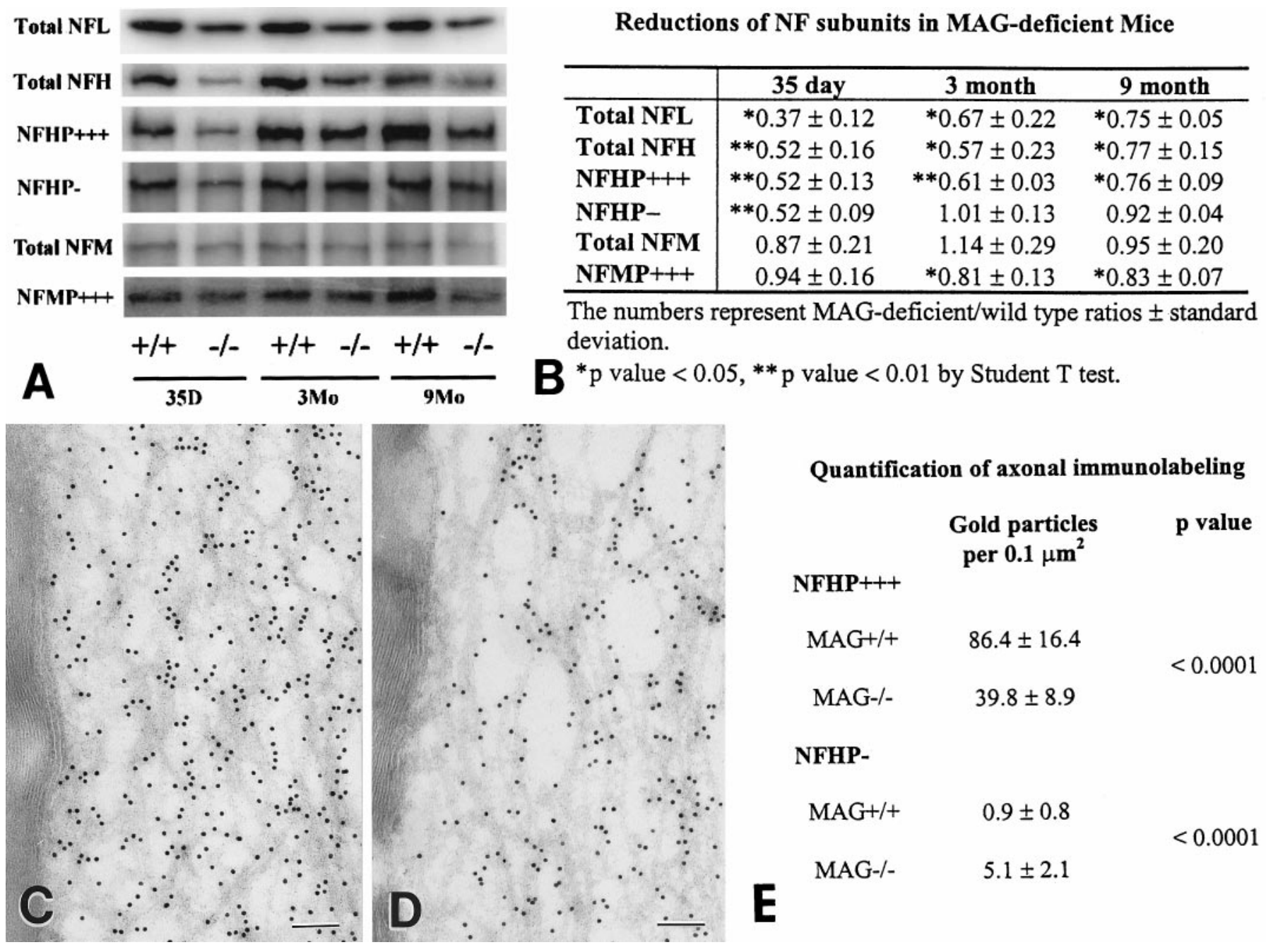

Figure 4. Neurofilament proteins and their phosphorylation states are reduced in sciatic nerves of MAG-deficient mice. $A$ shows comparison between quantitative Western blots of neurofilament epitopes in control $(+/+)$ and MAG-deficient $(-/-)$ sciatic nerve homogenates from P35 and 3- and 9-month old mice. NFHP -, Poorly or nonphosphorylated NFH; NFHP + + + and NFMP +++ , highly phosphorylated NFH and NFM. Three MAG-deficient and three control nerves were analyzed in triplicate at each time point, and representative bands were quantitated and expressed as MAG-deficient/wild-type ratios $\pm \mathrm{SD}(B)$. Total NFL, total NFH, and NFHP +++ were significantly reduced at all ages. NFHP- was reduced in 35-d-old MAG-deficient homogenates but not at later time points. Total NFM remained unchanged at all ages, whereas NFMP +++ was significantly reduced in 3- and 9-month-old MAG-deficient homogenates. $C-E$, Ultrathin cryosections of 9-month-old control $(C)$ and MAG-deficient $(D)$ sciatic nerves were immunostained with $\mathrm{NFHP}+++$ antibodies by immunogold procedures. Compared with control nerves $(C)$, MAG-deficient nerves $(D)$ contained a $50 \%$ decrease in $\mathrm{NFHP}+++$ labeling $(E)$. In contrast, NFHP- labeling was significantly increased in MAG-deficient nerves $(E)$. Scale bars, $0.1 \mu \mathrm{m}$.

Paranodal regions (57 and 93\%) from 3- and 9-month-old MAGdeficient mice had thick-appearing myelin segments (Fig. 5B). Compared with control fibers, segments of internodal thickappearing myelin were also increased in 3- and 9-month-old MAGdeficient mice (Fig. $5 B$ ), but they were less frequent than those in paranodal regions. In electron micrographs of MAG-deficient paranodes, the excessive myelin was positioned asymmetrically around axons with remarkably small calibers (Fig. 5C). Electron microscopic examination of longitudinal nerve sections established that small axonal calibers characteristic of nodes of Ranvier extended into paranodal regions of MAG-deficient fibers. Figure $5 D$ shows the dramatic difference between axonal caliber in nodal and paranodal regions in 9-month-old control nerve. In contrast, axonal calibers in 9-month-old MAG-deficient fibers did not increase significantly in paranodal regions that contained redundant myelin (Fig. 5E). Thick-appearing para-nodal myelin, therefore, is generated in part by axonal shrinkage and collapse of myelin.

\section{DISCUSSION}

This report establishes for the first time that MAG modulates caliber, neurofilament spacing, and neurofilament phosphorylation of myelinated axons. In addition, absence of MAG results in axonal atrophy and Wallerian degeneration of myelinated fibers. Axonal atrophy was most prominent in paranodal regions where it resulted in formation of tomaculi. Decreased axonal calibers, neurofilament spacing, and neurofilament phosphorylation have been described previously in peripheral nerves from Trembler mice (deWaegh et al., 1992). Trembler mice have a point mutation in the PMP22 gene that causes a dysmyelinating peripheral neuropathy (Suter et al., 1992) mediated by a "gain of function" of the mutated PMP22. The mechanism by which mutated PMP22 modulates axonal cytoskeleton and reduced axonal caliber is unclear, and the chronic demyelination and remyelination of Trembler axons complicates the interpretation of underlying 


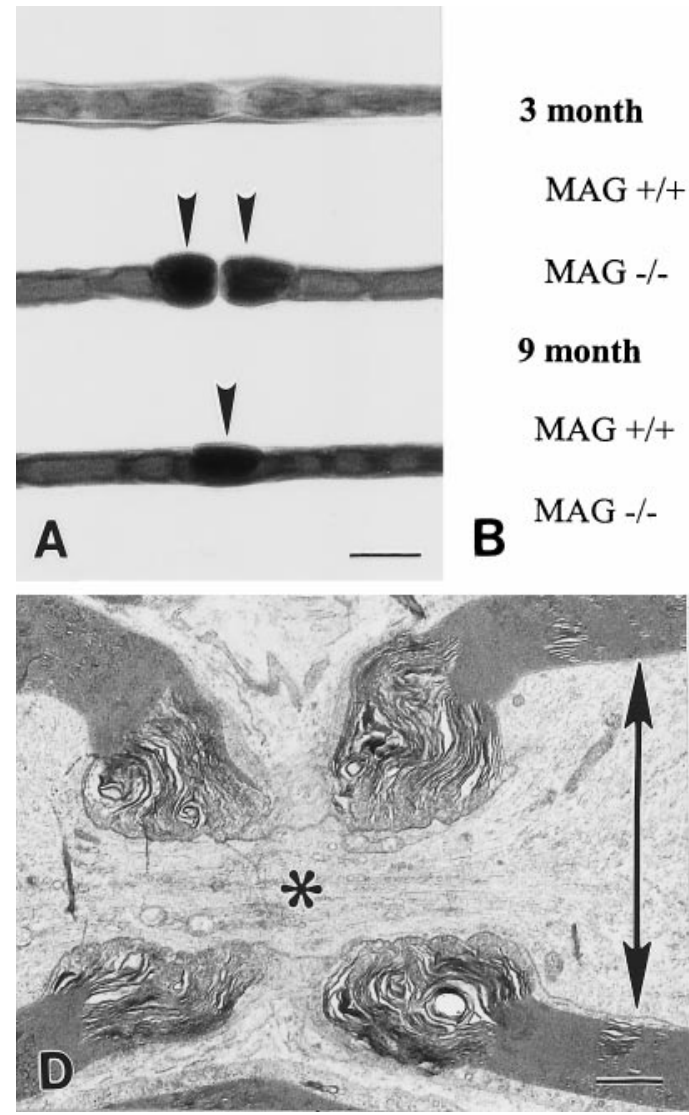
Hypermyelinated segment paranodal internodal

$7 / 170 \quad 2 / 85$

$98 / 170$

$23 / 85$ $<0.00001$ p value

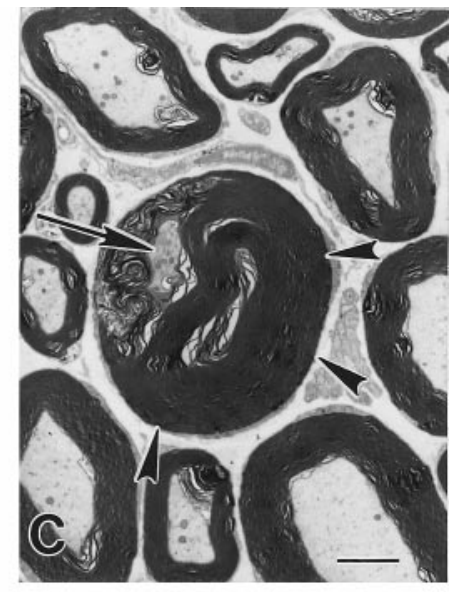

$6 / 150$

$278 / 300$

$123 / 150$

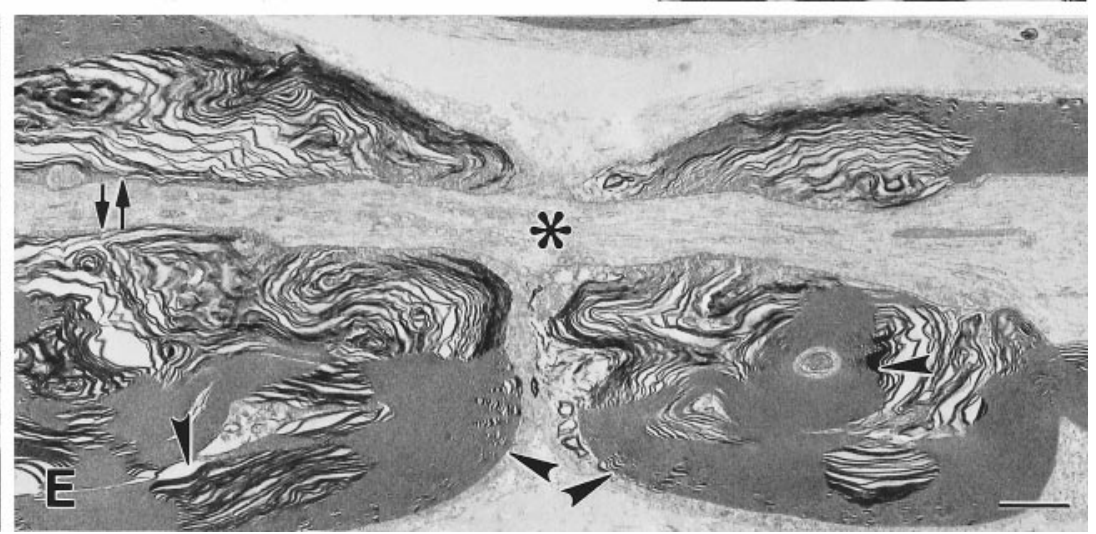

Figure 5. Tomaculi result from axonal shrinkage and myelin collapse. A, Teased fibers from 9-month-old control (top) and MAG-deficient (center and bottom) sciatic nerves. Thick paranodal myelin was rare in control fibers but abundant in MAG-deficient fibers (center, arrowheads). Internodal segments also had thick myelin in MAG-deficient fibers (bottom, arrowhead). Scale bar, $20 \mu \mathrm{m}$. B, Thick-appearing myelin segments were quantified, grouped by location (paranodal and internodal), and found at significantly greater numbers in MAG-deficient fibers when compared with control fibers. $C-E$, Electron micrographs of paranodal regions of MAG-deficient $(C, E)$ and control $(D)$ myelin internodes. In transverse section, thick redundant myelin $(C$, arrowheads) partially surrounds an axon $(C$, arrows) with remarkably small caliber. In longitudinal orientation, the difference between nodal $(*)$ and internodal (arrows) axonal caliber is much greater in control $(D)$ than in MAG-deficient $(E)$ fibers. Redundant paranodal myelin surrounds paranodal axons with reduced caliber in MAG-deficient fibers (E, arrowheads). Scale bars: $C, 2.0 \mu \mathrm{m} ; D, E, 1.0 \mu \mathrm{m}$.

mechanisms. Nevertheless, the striking similarities between our data and those described in Trembler mice raised the possibility that reductions in axonal caliber, neurofilament spacing, and neurofilament phosphorylation can be caused by two separate molecules (MAG and PMP22) that participate in a common pathway. In contrast to Trembler mice, axonal changes in MAGdeficient mice result from a "loss of function," and interpretation of this effect is not compounded by chronic hypomyelination, demyelination, and remyelination. In addition, near-normal calibers can be obtained when myelin-forming cells ensheath but fail to myelinate axons (Sanchez et al., 1996), supporting the notion that axonal calibers are regulated by a molecule in the adaxonal membrane of myelin-forming cells.

\section{Loss of MAG results in reduced neurofilament spacing}

How does MAG modulate axonal caliber? Reduced axonal calibers in MAG-deficient mice could, in principle, reflect decreased neurofilament numbers or spacings or both. Our results indicate that the primary determinant of reduced axon calibers in MAGdeficient mice is reduced neurofilament spacing. Furthermore, our data support the hypothesis that MAG affects phosphorylation of NFH and NFM. This is best supported by our quantitative immunolabeling studies in which highly phosphorylated NFH epitopes were reduced by $50 \%$. We sampled fibers that theoreti- cally should have had the largest axonal calibers based on their number of compact myelin lamella. A fivefold increase in nonphosphorylated NFH epitopes was also detected in sections from MAG-deficient nerves. Decreased NFH phosphorylation was also confirmed by Western blot analysis. Compared with control nerve homogenates, MAG-deficient nerves contained reduced $\mathrm{NFHP}+++$ levels relative to nonphosphorylated $\mathrm{NFH}$ and a $25 \%$ reduction in NFMP +++ epitopes. These data are consistent with previous reports that correlated reduced axonal caliber with reduced NF spacing and NF phosphorylation (deWaegh et al., 1992; Tu et al., 1995). If NF spacing is regulated by bulk NF phosphorylation, then one would predict greater reductions in $\mathrm{NFHP}+++$ and NFMP +++ than observed in our Western blot analysis. It is possible, therefore, that phosphorylation of select KSP or other sites regulates lateral extension of NF sidearms. Collectively, our data are most consistent with the hypothesis that the absence of MAG causes reduced phosphorylation of NFH and NFM, retraction of neurofilament sidearms, reduced neurofilament spacing, and smaller axonal calibers.

\section{Loss of MAG caused reduced neurofilament phosphorylation}

How does MAG regulate neurofilament spacing? The biochemical properties and periaxonal of location of MAG are consistent 
with the possibility that MAG regulates axonal cytoskeleton through interaction with an axolemmal receptor. In support of this function, MAG-containing liposomes adhere to neurons (Poltorak et al., 1987; Johnson et al., 1989), and MAG inhibits neurite extension (McKerracher et al., 1994; Mukhopadhyay et al., 1994) and can cause axonal growth cone collapse (Li et al., 1996). Neurofilament phosphorylation depends on a delicate balance between the activities of axonal kinases and phosphatases (Pant and Veeranna, 1995) that include glycogen synthase kinase $3 \alpha$ (Yang et al., 1995), cyclin-dependent kinase 5 (cdk5) (Shetty et al., 1993), and protein phosphatase 2a (Veeranna et al., 1995). Western blot analysis detected similar levels of cdk5 in homogenates of control and MAG-deficient sciatic nerve (X. Yin, unpublished observations). Kinase activity of cdk5 depends on the formation of a multimeric complex that has several activators (Lew et al., 1992). It is possible that MAG directly or indirectly initiates a signaling cascade that modulates kinase complexes or activators. It is also possible that the overall organization of axonal cytoskeleton may be compromised in MAG-deficient mice, and this may reduce neurofilament phosphorylation and neurofilament spacing by a more indirect mechanism. Although microtubule distributions were not studied extensively in this report, microtubule-enriched and microtubule-free regions were conspicuously apparent in electron micrographs of axons from MAG-deficient mice (Yin, unpublished observations). Because alteration in microtubules and axonal transport accompany reduced axonal calibers and neurofilament spacing in Trembler peripheral nerve (Kirkpatrick and Brady, 1994), similar studies may provide additional insights into the mechanisms of axonal atrophy in MAG-deficient mice.

Because neurofilaments are obligate heteropolymers, changes in total NF subunits or subunit ratios could also affect axonal caliber by changing neurofilament number and phosphorylation. Although total NFL and NFH subunits were reduced in MAG-deficient nerves, NF numbers were similar to those in control nerves. These findings suggest a smaller nonfilamentous NF subunit pool in MAG-deficient mice and support previous in vitro (Ching and Liem, 1993; Nakagawa et al., 1995) and in vivo (Veeranna et al., 1995; Xu et al., 1996) studies that demonstrate NF assembly over a wide range of NF subunit stoichiometries. The contribution of NF subunits to radial axonal growth has been studied by overexpressing each subunit or a combination of subunits in transgenic mice. One- to twofold increases in individual neurofilament subunit ratios inhibit radial growth of axons (Cote et al., 1993; Xu et al., 1996). The $25 \%$ increase in NFM/NFL ratios in 9-month-old MAG-deficient nerves could contribute, in principle, to reduce neurofilament spacing and axonal calibers.

\section{Loss of MAG causes paranodal tomaculi}

Regional variations in axonal calibers were prominent within individual myelin fibers of MAG-deficient mice. Most striking was a dramatic reduction of caliber and neurofilament spacing in paranodal axons. In MAG-deficient mice, paranodal axonal calibers expanded appropriately during early postnatal development. Between P35 and P90, paranodal axonal calibers in normal mice continue to expand, whereas those in MAG-deficient mice shrink. Reduction in paranodal axonal calibers resulted in paranodal tomaculi in $57 \%$ of fibers in 90-d-old MAG-deficient mice and in $95 \%$ of fibers in 9-month-old MAG-deficient mice. Tomaculi described in another line of MAG knockout mice (Carenini et al., 1997) and in heterozygous PMP22 knockout
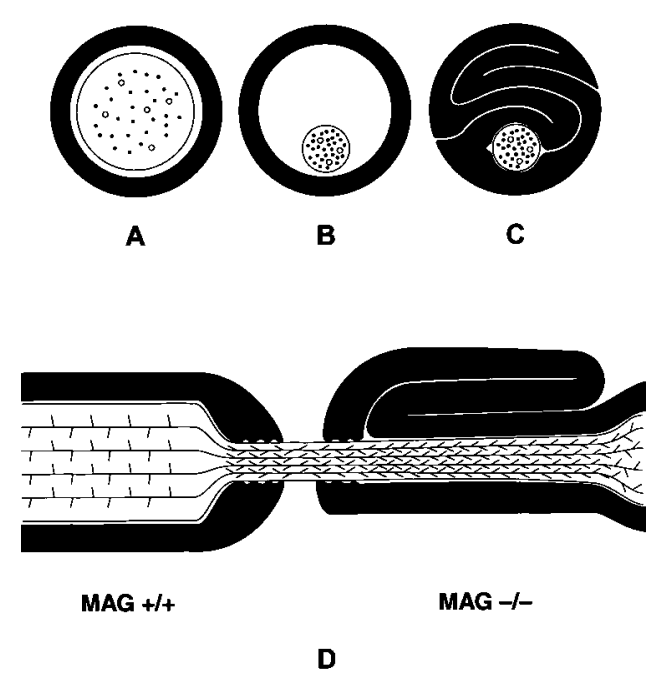

Figure 6. Schematic representation of tomaculi formation in MAGdeficient paranodal regions. $A-C$, Relationship between axonal caliber and myelin sheath in transverse section of normal myelinated fiber $(A)$. In MAG-deficient nerves, paranodal axonal calibers shrink $(B)$ and result in myelin sheath collapse and tomaculi formation $(C)$. $D$, In $\mathrm{MAG}+/+$ paranodal regions, increased neurofilament spacings correlate with large axonal caliber. In $\mathrm{MAG}-/-$ paranodal regions surrounded by tomaculi, neurofilament spacings and axonal calibers are similar to those in nodal regions.

mice (Adlkofer et al., 1997) were attributed to paranodal hypermyelination. The symmetrical distribution of redundant myelin around paranodal axons in heterozygous PMP22 mice (Adlkofer et al., 1997) supports hypermyelination as a cause of tomaculi formation. The dramatic reduction in paranodal axonal calibers and the asymmetrical distribution of the redundant myelin around the axon in MAG-deficient mice is more consistent with collapse of myelin that formed around larger axonal cylinders (Fig. 6).

Regional variations in axonal caliber of MAG-deficient mice raise several additional possibilities regarding mechanisms of Schwann cell-axon interactions. First, the role of MAG in modulating axonal cytoskeleton may differ based on location along the internode. Second, other Schwann cell molecules that are concentrated within or transported to paranodal regions (and possibly to axons) may affect neurofilament spacing and axonal caliber. Third, MAG helps segregate nodal axoplasmic environments that favor NF sidearm retraction (i.e., NF dephosphorylation) from paranodal environments that favor NF sidearm extension (i.e., NF phosphorylation). In chronic absence of MAG, these nodal components or microenvironments may diffuse into paranodal axoplasm and cause sidearm retraction.

\section{Summary}

This report has established that MAG-deficient mice develop a chronic atrophy of myelinated axons that can lead to axonal degeneration. Although the numbers of degenerating myelinated fibers in MAG-deficient nerves were small (two to nine per section), they were present in all sections examined from P35 and older mice, and were detected only in control nerves from 16month-old mice. Axonal atrophy and degeneration as a consequence of demyelination have been reported in multiple sclerosis brains (Trapp et al., 1998) and in chronic relapsing experimental allergic encephalomyelitis (Raine and Cross, 1989). MAGdeficient mice are an excellent model for investigating mecha- 
nisms of axonal atrophy and degeneration, and they provide a unique setting to test potential therapeutics that may delay or stop axonal atrophy associated with chronic diseases of myelin.

\section{REFERENCES}

Adlkofer K, Frei R, Neuberg DH-H, Zielasek J, Toyka KV, Suter U (1997) Heterozygous peripheral myelin protein 22-deficient mice are affected by a progressive demyelinating tomaculous neuropathy. J Neurosci 17:4662-4671.

Aguayo AJ, Epps J, Charron L, Bray GM (1976) Multipotentiality of Schwann cells in cross anastomosed and grafted myelinated and unmyelinated nerves: quantitative microscopy and radioautography. Brain Res 104:1-20.

Arbuthnott ER, Boyd IA, Kalu KU (1980) Ultrastructural dimensions of myelinated peripheral nerve fibres in the cat and their relation to conduction velocity. J Physiol (Lond) 308:125-157.

Arquint M, Roder J, Chia L-S, Down J, Wilkinson O, Bayley H, Braun P, Dunn R (1987) Molecular cloning and primary structure of myelinassociated glycoproteins. Proc Natl Acad Sci USA 84:600-604.

Carenini S, Montag D, Cremer H, Schachner M, Martini R (1997) Absence of the myelin-associated glycoprotein (MAG) and the neura cell adhesion molecule (N-CAM) interferes with the maintenance, but not with the formation of peripheral myelin. Cell Tissue Res 287:3-9.

Ching GY, Liem RK (1993) Assembly of type IV neuronal intermediate filaments in non-neuronal cells in the absence of pre-existing cytoplasmic intermediate filaments. J Cell Biol 122:1323-1335.

Cote F, Collard JF, Julien JP (1993) Progressive neuronopathy in transgenic mice expressing the human neurofilament heavy gene: a mouse model of amyotrophic lateral sclerosis. Cell 73:35-46.

deWaegh SM, Lee VM-Y, Brady ST (1992) Local modulation of neurofilament phosphorylation, axonal caliber, and slow axonal transport by myelinating Schwann cells. Cell 68:451-463.

Eyer J, Peterson A (1994) Neurofilament-deficient axons and perikaryal aggregates in viable transgenic mice expressing a neurofilament- $\beta$ galactosidase fusion protein. Neuron 12:389-405.

Friede RL, Samorajski T (1971) Axonal caliber related to neurofilaments and microtubules in sciatic nerve fibers of rats and mice. Anat Rec 167:379-387.

Fruttiger M, Montag D, Schachner M, Martini R (1995) Crucial role for the myelin-associated glycoprotein in the maintenance of axon-myelin integrity. Eur J Neurosci 7:511-515.

Griffin JW, Kidd G, Trapp BD (1993) Interactions between axons and Schwann cells. In: Peripheral neuropathy (Dyck PJ, Thomas PK, eds), pp 317-330. Philadelphia: Saunders.

Hirokawa N, Glicksman MA, Willard MB (1984) Organization of mammalian neurofilament polypeptides within the neuronal cytoskeleton. $\mathrm{J}$ Cell Biol 98:1523-1536.

Hoffman PN, Griffin JW (1992) Regulation of axonal caliber. In: Peripheral neuropathy (Dyck PJ, Thomas PK, Griffin JW, Low PA, Poduslo J, eds), pp 389-402. New York: Raven.

Hoffman PN, Lasek RJ (1975) The slow component of axonal transport: identification of major structural polypeptides of the axon and their generality among mammalian neurons. J Cell Biol 66:351-366.

Hsieh S-T, Crawford TO, Bouldin TW, Griffin JW (1993) Influence of demyelination and remyelination on axonal organization. Brain Pathol 3:307.

Hsieh S-T, Kidd GJ, Crawford TO, Xu Z, Trapp BD, Cleveland DW, Griffin JW (1994) Regional modulation of neurofilament organization by myelination in normal axons. J Neurosci 14:6392-6401.

Johnson PW, Abramow-Newerly W, Seilheimer B, Sadoul R, Tropak MB, Arquint M, Dunn RJ, Schachner M, Roder JC (1989) Recombinant myelin-associated glycoprotein confers neural adhesion and neurite outgrowth function. Neuron 3:377-385.

Kirkpatrick LL, Brady ST (1994) Modulation of the axonal microtubule cytoskeleton by myelinating Schwann cells. J Neurosci 12:7440-7450.

Lai C, Brow MA, Nave K-A, Noronha AB, Quarles RH, Bloom FE, Milner RJ, Sutcliffe JG (1987) Two forms of 1B236/myelin-associated glycoprotein (MAG), a cell adhesion molecule for postnatal neural development, are produced by alternative splicing. Proc Natl Acad Sci USA 84:4337-4341.

Lew J, Winkfein RJ, Paudel HK, Wang JH (1992) Brain prolinedirected protein kinase is a neurofilament kinase which displays high sequence homology to p34 ${ }^{\text {cdc2 }}$. J Biol Chem 267:25922-25926.
Li C, Tropak MB, Gerial R, Clapoff S, Abramow-Newerly W, Trapp B, Peterson A, Roder J (1994) Myelination in the absence of myelinassociated glycoprotein. Nature 369:747-750.

Li M, Shibata A, Li C, Braun PE, McKerracher L, Roder J, Kater SB, David S (1996) Myelin-associated glycoprotein inhibits neurite/axon growth and causes growth cone collapse. J Neurosci Res 46:404-414.

McKerracher L, David S, Jackson DL, Kottis V, Dunn RJ, Braun PE (1994) Identification of myelin-associated glycoprotein as a major myelin-derived inhibitor of neurite growth. Neuron 13:805-811.

Montag D, Giese KP, Bartsch U, Martini R, Land Y, Blüthmann H, Karthigasan J, Kirschner DA, Wintergerst ES, Nave K-A, Zielasek J, Toyka KV, Lipp H, Schachner M (1994) Mice deficient for the myelinassociated glycoprotein show subtle abnormalities in myelin. Neuron 13:229-246.

Mukhopadhyay G, Doherty P, Walsh FS, Crocker PR, Filbin MT (1994) A novel role for myelin-associated glycoprotein as an inhibitor of axonal regeneration. Neuron 13:757-767.

Nakagawa T, Chen J, Zhang Z, Kanai Y, Hirokawa N (1995) Two distinct functions of the carboxyl-terminal tail domain of NF-M upon neurofilament assembly: cross-bridge formation and longitudinal elongation of filaments. J Cell Biol 129:411-429.

Ohara O, Gahara Y, Miyake T, Teraoka H, Kitamura T (1993) Neurofilament deficiency in quail caused by nonsense mutation in neurofilament-L gene. J Cell Biol 121:387-395.

Owens GC, Bunge RP (1991) Schwann cells infected with a recombinant retrovirus expressing myelin-associated glycoprotein antisense RNA do not form myelin. Neuron 7:565-575.

Owens GC, Boyd CJ, Bunge RP, Salzer JL (1990) Expression of recombinant myelin-associated glycoprotein in primary Schwann cells promotes the initial investment of axons by myelinating Schwann cells. J Cell Biol 111:1171-1182.

Pant HC, Veeranna (1995) Neurofilament phosphorylation. Biochem Cell Biol 73:575-592.

Poltorak M, Sadoul R, Keilhauer G, Landa C, Fahrig T, Schachner M (1987) Myelin-associated glycoprotein, a member of the L2/HNK-1 family of neural cell adhesion molecules, is involved in neuronoligodendrocyte and oligodendrocyte-oligodendrocyte interaction. J Cell Biol 105:1893-1899.

Raine CS, Cross AH (1989) Axonal dystrophy as a consequence of long-term demyelination. Lab Invest 60:714-725.

Salzer JL, Holmes WP, Colman DR (1987) The amino acid sequences of the myelin-associated glycoproteins: homology to the immunoglobulin gene superfamily. J Cell Biol 104:957-965.

Sanchez I, Hassinger L, Paskevich PA, Shine HD, Nixon RA (1996) Oligodendroglia regulate the regional expansion of axon caliber and local accumulation of neurofilaments during development independently of myelin formation. J Neurosci 16:5095-5105.

Shetty KT, Link WT, Pant HC (1993) cdc2-like kinase from rat spinal cord specifically phosphorylates KSPXK motifs in neurofilament proteins: isolation and characterization. Proc Natl Acad Sci USA 90:6844-6848.

Sternberger NH, Quarles RH, Itoyama Y, Webster HdeF (1979) Myelinassociated glycoprotein demonstrated immunocytochemically in myelin and myelin-forming cells of developing rats. Proc Natl Acad Sci USA $76: 1510-1514$.

Suter U, Welcher AA, Ozcelik T, Snipes GJ, Kosaras B, Francke U, Billings-Gagliardi S, Sidman RL, Shooter EM (1992) Trembler mouse carries a point mutation in a myelin gene. Nature 356:241-244.

Trapp BD, Quarles RH (1982) Presence of the myelin-associated glycoprotein correlates with alterations in the periodicity of peripheral myelin. J Cell Biol 92:877-882.

Trapp BD, Andrews SB, Cootauco C, Quarles RH (1989) The myelinassociated glycoprotein is enriched in multivesicular bodies and periaxonal membranes of actively myelinating oligodendrocytes. J Cell Biol 109:2417-2426.

Trapp BD, Peterson J, Ransohoff RM, Rudick R, Mörk S, Bö L (1998) Axonal transection in multiple sclerosis lesions. N Engl J Med 338:278-285.

Tu P-H, Elder G, Lazzarini RA, Nelson D, Trojanowski JQ, Lee VM-Y (1995) Overexpression of the human NFM subunit in transgenic mice modifies the level of endogenous NFL and the phosphorylation state of NFH subunits. J Cell Biol 129:1629-1640. 
Veeranna, Shetty KT, Link WT, Jaffe H, Wang J, Pant HC (1995) Neuronal cyclin-dependent kinase-5 phosphorylation sites in neurofilament protein (NF-H) are dephosphorylated by protein phosphatase 2A. J Neurochem 64:2681-2690.

Waxman SG (1980) Determinants of conduction velocity in myelinated nerve fiber. Muscle Nerve 3:141-150.

Weinberg HJ, Spencer PS (1976) Studies on the control of myelinogenesis. II. Evidence for neuronal regulation of myelin production. Brain Res 113:363-378.

Windebank AJ, Word P, Bunge RP, Dyck PJ (1985) Myelination determines the caliber of dorsal root ganglion neurons in culture. J Neurosci 5:1563-1567.
Wong PC, Marszalek J, Crawford TO, Xu Z, Hsieh S-T, Griffin JW, Cleveland DW (1995) Increasing neurofilament subunit NF-M expression reduces axonal NF-H, inhibits radial growth, and results in neurofilamentous accumulation in motor neurons. J Cell Biol 130:1413-1422.

Xu Z, Marszalek JR, Lee MK, Wong PC, Folmer J, Crawford TO, Hsieh S-T, Griffin JW, Cleveland DW (1996) Subunit composition of neurofilaments specifies axonal diameter. J Cell Biol 133:1061-1069.

Yang SD, Huang JJ, Huang TJ (1995) Protein kinase $\mathrm{F}_{\mathrm{A}}$ /glycogen synthase kinase 3 alpha predominantly phosphorylates the in vivo sites of Ser502, Ser506, Ser603, and Ser666 in neurofilament. J Neurochem 64:1848-1854. 